Research Paper

\title{
Gene Expression Profiling in Winged and Wingless Cotton Aphids, Aphis gossypii (Hemiptera: Aphididae)
} \author{
Qingwen Zhang ${ }^{\circledR}$, Xuguo Zhou ${ }^{\circledR}$ \\ 1. Department of Entomology, China Agricultural University, Beijing 100193, China. \\ 2. Department of Entomology, University of Kentucky, Lexington, KY 40546-0091, USA. \\ * These authors contributed equally to this research.
}

Xiaowei Yang ${ }^{1 *}$, Xiaoxia Liu ${ }^{1 *}$, Xiangli $\mathrm{Xu}^{1}$, Zhen $\mathrm{Li}^{1}$, Yisong $\mathrm{Li}^{1}$, Dongyan Song ${ }^{2}$, Tian $\mathrm{Yu}^{2}$, Fang Zhu ${ }^{2}$

$\triangle$ Corresponding author: Dr. Qingwen Zhang, Department of Entomology, China Agricultural University, Beijing, China, 100193. Phone: +86-10-6273-3016 Fax: +86-10-6273-3016 Email: zhangqingwen@263.net. Or Dr. Xuguo "Joe" Zhou, Department of Entomology, University of Kentucky, S-225 Agricultural Science Centre North, Lexington, KY, USA 40546-0091. Phone: +1- 859-257-3125 Fax: +1-859-323-1120 Email: xuguozhou@uky.edu.

(C) Ivyspring International Publisher. This is an open-access article distributed under the terms of the Creative Commons License (http://creativecommons.org/ licenses/by-nc-nd/3.0/). Reproduction is permitted for personal, noncommercial use, provided that the article is in whole, unmodified, and properly cited.

Received: 2013.09.10; Accepted: 2014.0I.22; Published: 2014.02.19

\begin{abstract}
While trade-offs between flight capability and reproduction is a common phenomenon in wing dimorphic insects, the molecular basis is largely unknown. In this study, we examined the transcriptomic differences between winged and wingless morphs of cotton aphids, Aphis gossypii, using a tag-based digital gene expression (DGE) approach. Ultra high-throughput Illumina sequencing generated 5.30 and 5.39 million raw tags, respectively, from winged and wingless A. gossypii DGE libraries. We identified 1,663 differentially expressed transcripts, among which 58 were highly expressed in the winged $A$. gossypii, whereas 1,605 expressed significantly higher in the wingless morphs. Bioinformatics tools, including Gene Ontology, Cluster of Orthologous Groups, euKaryotic Orthologous Groups and Kyoto Encyclopedia of Genes and Genomes pathways, were used to functionally annotate these transcripts. In addition, 20 differentially expressed transcripts detected by DGE were validated by the quantitative real-time PCR. Comparative transcriptomic analysis of sedentary (wingless) and migratory (winged) A. gossyii not only advances our understanding of the trade-offs in wing dimorphic insects, but also provides a candidate molecular target for the genetic control of this agricultural insect pest.
\end{abstract}

Key words: Aphis gossypii, trade-off, migration, digital gene expression, wing polyphenism.

\section{Introduction}

Trade-offs between life-history traits have been studied in a variety of organisms [1-3]. Individuals capable of fly benefit from the ability, but it is also costly on energy for flight mechanism and flight behavior. This energy, alternatively, can be used for other aspects, such as body size and reproduction [4]. In the past forty years, trade-offs between flight and reproduction has been widely studied [5-9]. Wing dimorphic insects have been studied extensively and have emerged as a great model system for the study of trade-offs in the past several decades [8-10]. Wing polyphenism has been observed in a wide range of insect species, including Hemiptera, Orthoptera, and Coleoptera, to facilitate their range expansion [8, 10-12]. Wing dimorphic insects display commonly dispersing and non-dispersing morphs [13, 14]. Flight morphs, with long wings, flight muscles and flight capability, need additional energy and time for the migratory flight. Flightless morphs, with reduced or absent wings and underdeveloped flight muscles, show an earlier onset of oviposition and enhanced reproductive output [8, 15-17]. Trade-offs have been 
studied at behavioral, morphological, physiological and biochemical levels in wing dimorphic insects, such as crickets, migratory locusts, and aphids $[16,18$, 19]. For instance, biochemical aspects and intermediary metabolisms are investigated in a wing dimorphic cricket, Gryllus firmus [20-22]. However, the genetic basis of biochemistry and metabolism related to trade-offs remains unclear.

There are trade-offs related to dispersal and reproduction in aphids between two wing morphs. The wing polyphenism in aphids is well-studied [23, 24]. Winged and wingless aphids exist in both sexual and parthenogenetic stages of its life cycle. The migrants in spring, summer and early autumn are parthenogenetic generations, and the migration normally occurs in spring and summer [25, 26]. Wing polyphenism occurs primarily among parthenogenetic females, whereas wing polymorphism, which is genetically determined, has been found only in males [14, 24]. Parthenogenetic females with different wing morphs are descended from the same ancestry. A single genotype can produce various phenotypes as a result of exposure to various environmental conditions [27]. Such phenotypic plasticity is one of the main reasons contributing to the ecological success of aphids and their increasing pest status [26, 28]. Plasticity is not only an adaptive behavioral strategy that has evolved in most insect orders for coping with complex and uncertain environments, but also a key factor that leads to population expansion and infestation. Wing-polyphenic aphids exhibited distinct morphological differences between two wing types of aphids, and showed differences in their life history, particularly in their behavioral and physiological traits [14, $29,30]$. The biochemical basis of wing polyphenism related to energy resources, including glycogen, trehalose, and lipids, was investigated in cotton aphid, Aphis gossypii [30] and grain aphid, Sitobion avenae [31]. Total lipid and triglyceride were significantly higher in winged morphs, whereas glycogen, soluble protein and water were higher in wingless morphs in both species. Recent genetic studies [32-34], especially the release of a draft genome of pea aphid Acyrthosiphon pisum [35], lays the foundation for a better understanding of trade-offs between flight and reproduction at the molecular level. Genome-wide transcriptome analyses provide a new avenue for the future functional characterization study.

Digital gene expression (DGE) is a tag-based RNA sequencing approach that allows millions of RNA reads, including differentially expressed transcripts, to be identified in a sample with or without any annotation information [36]. For aphid wing polyphenism study, DGE has several advantages - a greater sequencing depth, detection of unknown transcripts, and sensitivity [37]. Although external factors such as environmental cues, population density and nutrition status have been studied for years, the genetic basis of trade-offs in the cotton aphids, $A$. gossypii, a major cotton pest worldwide, has yet been examined in details. The objectives of this study are 1) to reveal differential gene expression profiles between winged and wingless $A$. gossypii adults, and 2) to establish a link between transcriptomic profiles and biochemical differences observed in previous studies. Our studies provide molecular basis that underpins trade-offs between flight capability and reproduction in A. gossypii and will facilitate the sustainable cotton aphid management through the RNA interference-mediated genetic disruption of its migratory behavior.

\section{Materials and Methods}

\section{Aphis gossypii maintenance and sample preparation}

A single A. gossypii female adult was collected from a cotton field $\left(40^{\circ} 01^{\prime} \mathrm{N}, 116^{\circ} 17^{\prime} \mathrm{E}\right)$ at the China Agricultural University, Beijing, China, in July of 2009. Aphid colonies generated from this individual A. gossypii had been established in the laboratory for more than one year before being sacrificed in the subsequent experiments. Specifically, parthenogenetic adults were provisioned with cotton seedlings (three-leaf stage, cultivar: Zhong 35) and maintained in a growth chamber at $60 \%$ relative humidity, and 16 : $8 \mathrm{~L}$ : $\mathrm{D}$ photoperiod with $24 \pm 1^{\circ} \mathrm{C}$ in the light and $22 \pm 1^{\circ} \mathrm{C}$ in the dark [30], [34,38]. Winged forms were induced by crowding in cotton leaves two weeks after A. gossypii was transferred to new host plants. The fourth instars were placed into individual dishes, and observed daily until molting. A total of 50 winged or wingless A. gossypii adults were collected separately within two days after molting. Aphids were frozen in liquid nitrogen immediately after the collection. RNA was extracted using the RNeasy Mini kit (Qiagen). Genomic DNA was digested by RNase-Free DNase Set (Qiagen). The quality and quantities of RNAs were determined using a Nanodrop2000 and Agilent bioanalyzer (Agilent).

\section{DGE library preparation and sequencing}

High-throughput DGE was performed by Solexa/Illumina sequencing technology at the Beijing Genomics Institute, Shenzhen, China. DGE libraries (winged and wingless) were prepared using the Illumina Gene Expression Sample Prep Kit. Briefly, poly(A) ${ }^{+}$RNA was enriched from $6 \mu \mathrm{g}$ of total RNA using oligomagnetic beads. Double-stranded cDNAs were directly synthesized on the poly $(\mathrm{A})^{+}$ 
RNA-bound beads and then digested with a 4 base recognition enzyme NlaIII. The fragmentized cDNAs were purified from the magnetic beads, containing $3^{\prime}$ ends and a sticky end. Then the Illumina adaptor 1 (sense: 5' ACACTCTTTCCCTACACGACGCTCTTCC GATC3') was ligated to the $5^{\prime}$ ends of these cDNA fragments. Digestion of MmeI and ligation of the Illumina adaptor 2 (sense: 5'GATCGGAAGAGCGGT TCAGCAGGAATGCCGAG3') produced short DNA fragments, with two adaptors and $21 \mathrm{bp}$ tags started with the CATG sites. Later on, Primer GX1 and Primer GX2 were added for PCR. After 15 cycles of linear PCR amplification, 95bp fragments were purified using 6\% TBE PAGE Gel electrophoresis. After denaturation, the single-stranded molecules were attached to the sequencing chip for sequencing via Illumina HiSeq ${ }^{\mathrm{TM}} 2000$ System. During this process, adaptor 1 was used as sequencing primer. Each tunnel of chip (flow cell) generated millions of raw tags with sequencing length of $35 \mathrm{bp}[39,40]$.

\section{Mapping of DGE tags}

Clean tags were generated by removing $3^{\prime}$ adaptor sequences, empty reads (with only $3^{\prime}$ adaptor sequences), low quality tags (tags with unknown nucleotide " $\mathrm{N}$ "), tags with erratic lengths, and tags with copy number of 1 . A total of 88,851 A. gossypii ESTs were selected at NCBI on Oct, 28, 2010. After eliminating low quality sequences and Phrap assembly (minmatch: 42; minscore: 40, repeat_stringency:0.96), 23,630 unigenes were obtained and used as reference dataset (Supplementary Material: Table S1). Clean tags were aligned to the reference dataset with only one nucleotide mismatch tolerance. The number of unambiguous clean tag corresponding to each transcript was counted and normalized to TPM (number of transcripts per million clean tags) [36, 41].

\section{Identification of differentially expressed transcripts}

The differentially expressed transcripts between winged and wingless patterns were identified using an algorithm developed by Audic and Claverie [42]. P value corresponds to differential gene expression test. FDR (False Discovery Rate) is a method of determining the threshold of $\mathrm{P}$ Value in multiple tests and analysis through manipulating FDR value [43]. In this study, we considered a transcript as differentially expressed when there was at least a 2 -fold difference in expression between the two samples with a FDR value $\leq 0.001$.

To functionally annotate these unigenes, distinct sequences were searched by BLASTx against the NCBI non-redundant protein database [44] with a cut-off E-value of $10^{-5}$, and assigned Cluster of
Orthologous Groups (COG) and euKaryotic Orthologous Groups (KOG) protein classes with rpstblastn algorithm against COG and KOG databases in NCBI (E-value $\leq 10^{-5}$ ) $[45,46]$. The BLASTx result was imported into Blast2GO software (version 2.6.4) to map the Gene Ontology (GO) categories [47], and the Kyoto Encyclopedia of Genes and Genomes (KEGG) pathways [48]. The enriched GO terms were developed by Blast2Go using Fisher's Exact Test with Multiple Testing Correction (FDR <0.05)[47]. The enrichment analysis of pathway was performed to further understand genes biological functions.

\section{qRT-PCR analysis}

PCR reactions were performed in a $20 \mu \mathrm{l}$ final volume containing $2 \times$ Power SYBR Green PCR Master Mix buffer (Applied Biosystems), $1 \mu \mathrm{M}$ of each specific primer and $1 \mu \mathrm{l}$ of diluted cDNA. PCR amplification was performed on an ABI Prism 7300 Sequence Detection System (Applied Biosystems) under the following conditions: $10 \mathrm{~min}$ at $95^{\circ} \mathrm{C}$ for polymerase activation, and 40 cycles of $95^{\circ} \mathrm{C}$ for $15 \mathrm{~s}, 60^{\circ} \mathrm{C}$ for $30 \mathrm{~s}$, and $72^{\circ} \mathrm{C}$ for 30 s for amplification signal collection. A default melting curve was produced to confirm single gene-specific peaks without primer dimerization present. Housekeeping genes, $\beta$-actin and $18 S$ rRNA were subjected to the reference gene selection experiment. Eventually, $\beta$-actin was used as a constitutive control to normalize the expressions of target genes using a $-\Delta \Delta$ Ct method.

To validate DGE results, a total of 20 transcripts were chosen from the top 10 differentially expressed transcripts of winged and wingless libraries, respectively, and three significantly enriched pathways. The gene-specific primers designed for reference and target genes were listed in Supplementary Material: Table S2. Three independent replications were carried out for the qRT-PCR validation study, with approximately 30 winged or wingless females for each replication.

\section{Results}

\section{Summary of digital gene expression sequencing}

Reference sequences were obtained from 88,851 ESTs, of which 36 low quality (less than 100bp after remove vector) sequences were discarded. 23,630 unigenes, containing 12,507 contigs and 11,123 singletons, with a mean length of $715 \mathrm{bp}$, were assembled by Phrap. The generated unigenes were subjected to BLAST search and functional annotation using various databases.

In total, 5.30 and 5.39 million raw tags were obtained from winged and wingless adults, respectively. 
Distribution details of tags were shown in Supplementary Material: Figure S1. Clean tags were mapped to 9,011 and 10161 unigenes, respectively (Table 1). Sequencing saturation analysis shows the number of detected genes almost ceases to increase when sequencing amount (total tag number) reaches 2 million (Supplementary Material: Figure S2), consequently, 5 million tags are sufficient for the expression profiling of cotton aphid. The total number of tags sequenced in the two samples was $10,479,972$. Tags with the same sequence were identified as a distinct tag, and their distributions in different wing morphs were documented. Tags in the two wing forms were compared in total and in distinct (Figure 1). Surprisingly, morph-specific tags (tags detected in one morph only) accounted for a very small proportion $(0.85 \%$ in winged morph, $2.12 \%$ in wingless morph) of the total clean tags, while the percentage of morph-specific tags increased obviously among the distinct tags.

\section{Differentially expressed transcripts in winged and wingless aphids}

We obtained 1,663 differentially expressed transcripts from a total of 8,308 tag-mapped unigenes. Among them, 58 transcripts were expressed significantly higher in winged aphids, while 1,605 transcripts had a higher expression in wingless morphs ( $\mid \log _{2}$ ratio $\mid \geq 1$; FDR value $\left.\leq 0.001\right)$. A total of 334 $(20.69 \%)$ differentially expressed transcripts had a differential ratio higher than $2\left(\mid \log _{2}\right.$ ratio $\mid \geq 2$; Figure $2)$, in which 3 transcripts were detected in winged forms only and 34 transcripts were expressed in wingless forms exclusively (Supplementary Material: Table S3). Top ten differentially expressed transcripts and their ratios are summarized in Table 2.
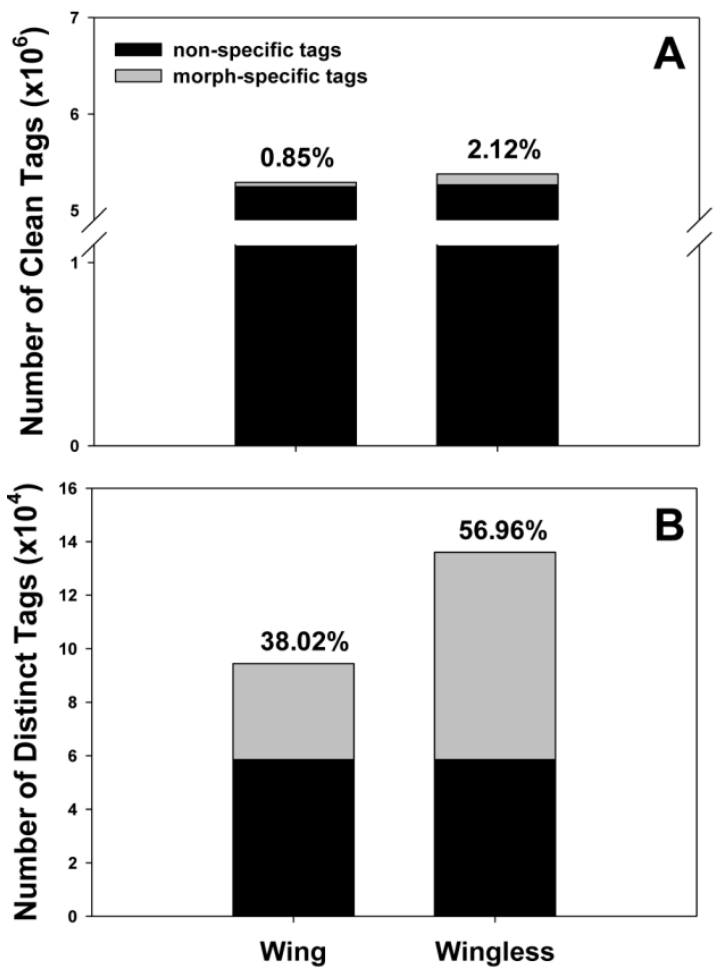

Figure I. Morph-specific sequencing tags in the winged and wingless $A$. gossypii. Number and percentage of morph-specific tags were documented in total clean tags (A) and distinct clean tags (B), respectively.



Figure 2. Expression dynamics of differentially expressed transcripts between winged and wingless $A$. gossypii. Fold Change represents the $\log _{2}$ of the ratio of gene expression level in winged in relation to wingless adult females. Transcript expressed at a constant level (ratio of I) has a log2 (ratio) of zero, which can be interpreted as "equally expressed among winged and wingless aphids". Positive number represents highly expressed transcripts in winged adult females. Negative number represents highly expressed transcripts in wingless adult females. 
Table I. Sequencing summary.

\begin{tabular}{|c|c|c|c|c|c|c|c|c|}
\hline \multirow[t]{2}{*}{ Sequence } & \multicolumn{4}{|c|}{ Winged Morph } & \multicolumn{4}{|c|}{ Wingless Morph } \\
\hline & Total & $\%$ & Distinct & $\%$ & Total & $\%$ & Distinct & $\%$ \\
\hline Clean Tag & 5221975 & - & 44414 & - & 5257997 & - & 61655 & - \\
\hline Mapped to gene & 4781916 & 91.57 & 21675 & 48.80 & 4345336 & 82.64 & 27998 & 45.41 \\
\hline Unambiguous tag & 1329628 & 25.4 & 20748 & 46.71 & 2322979 & 44.18 & 26749 & 43.38 \\
\hline Unknown tag & 440059 & 8.4 & 22739 & 51.20 & 912661 & 17.36 & 33657 & 54.59 \\
\hline
\end{tabular}

Table 2. Top ten differentially expressed transcripts between winged and wingless A. gossypii.

\begin{tabular}{lll}
\hline Gene ID & $\log _{2} \mathrm{FC}^{*}$ & Description \\
\hline AG10278 & 12.5 & Flightin-like \\
gi | 289097293 & 11 & 3-ketoacyl-CoA thiolase, mitochondrial-like \\
AG4984 & 10.1 & MPA13 allergen-like \\
gi | 289151497 & 8.3 & N/A \\
AG12533 & 8.1 & Phosphate carrier protein, mitochondrial-like \\
AG13078 & 5.7 & Adenine nucleotide translocator-like \\
AG6703 & 5.6 & PXMP2/4 family protein 4-like \\
AG11738 & 5.3 & Tectonin beta-propeller repeat-containing protein-like \\
AG12565 & 3.8 & Hypothetical protein LOC100165045 isoform 1 \\
AG11798 & 3.5 & Very long-chain specific acyl-CoA dehydrogenase \\
AG10938 & -9.7 & Seryl-tRNA synthetase, cytoplasmic-like \\
AG2471 & -9.3 & Hypothetical protein LOC100571294 \\
gi | 289129407 & -9.2 & N/A \\
AG9276 & -9.1 & RNA-binding protein PNO1-like \\
AG11819 & -9 & T-complex protein 1 subunit alpha-like \\
gi| 289076469 & -9 & N/A \\
AG7395 & -8.9 & Exosome complex exonuclease MTR3-like \\
gi | 289138983 & -8.7 & N/A \\
AG4684 & -8.6 & N/A \\
AG3568 & -8.6 & N/A \\
\hline “*": FC is the fold change of gene expression level between winged and wingless morphs. Positive and negative values, respectively, denote highly and lowly expressed gene \\
expression in winged adult females relative to wingless individuals. Assembly details are provided in Supplementary Material: Table S3.
\end{tabular}
expression in winged adult females relative to wingless individuals. Assembly details are provided in Supplementary Material: Table S3.

\section{Functional analysis of differentially expressed transcripts}

Following assignment of BLASTx top hits, 1,306 differentially expressed transcripts were functionally annotated with four additional databases. Among 8,308 sequenced transcripts, 3,184 transcripts were annotated with GO terms, including 2,318 involved in biological process, 2,547 in molecular function and 1,954 in cellular component. As to 1,663 differentially expressed transcripts, 763 transcripts were assigned with 3,168 GOs and can be classified into 42 secondary level categories (Figure 3). The category of biological process consists of 573 transcripts at 22 secondary levels. Molecular function category has 619 transcripts at 9 secondary levels. Cellular component includes 491transcripts in 11 secondary categories.

All differentially expressed transcripts were enriched in 16 GO group when compared to all the $A$. gossypii unigenes (Supplementary Material: Table S4). 7 of them were very deep level (specific) GO terms (Figure 4). Only one term (membrane) was underrepresented. In addition, transcripts were annotated by COG and KOG databases, by which 676 transcripts were categorized into 23 COG functional groups (Figure 5) and 1,144 transcripts into $25 \mathrm{KOG}$ classes (Figure 5). With KOG classification, in addition to the category of general function prediction only, the number of transcripts in category A (RNA processing and modification), $\mathrm{O}$ (Posttranslational modification, protein turnover and chaperones), and $\mathrm{U}$ (Intracellular trafficking, secretion, and vesicular transport) were 802, 748 and 759, respectively, higher than the number in the other categories.

Pathway analysis of differentially expressed transcripts was performed using the Kyoto Encyclopedia of Genes and Genomes (KEGG) database. Among 1,663 differentially expressed transcripts, 878 transcripts could be annotated in $\mathrm{KO}$ based on sequence homologies and mapped to 211 KEGG pathways (Table 3). Pathway-oriented analysis showed that the top three significantly enriched metabolic pathways in DGEs were ribosome, pyruvate metabolism and proteasome. Differentially expressed transcripts were also enriched in pathways relating to protein synthesis and degradation, lipid metabolism, immunity, RNA transport, and some signaling pathways. 


\section{qRT-PCR validation study}

qRT-PCR analysis was carried out to validate differentially expressed transcripts identified by DGE. All 20 genes showed the similar expression pattern as indicated by the DGE analysis (Table 4), suggesting that DGE approach can be a powerful tool for the discovery of candidate genes in a high-throughput manner.

Table 3. Top ten enriched metabolic pathways among differentially expressed transcripts.

\begin{tabular}{|c|c|c|c|c|c|}
\hline Pathway ID & Description & $\mathrm{DET}^{\mathrm{a}}$ & Reference $^{b}$ & P-value ${ }^{c}$ & Q-value \\
\hline ko03010 & Ribosome & 49 & 144 & 1.35E-14 & $2.85 \mathrm{E}-12$ \\
\hline ko00620 & Pyruvate metabolism & 21 & 83 & $8.96 \mathrm{E}-05$ & $9.45 \mathrm{E}-03$ \\
\hline ko03050 & Proteasome & 15 & 53 & 0.000239 & $1.68 \mathrm{E}-02$ \\
\hline ko03040 & Spliceosome & 44 & 268 & 0.001539 & 7.15E-02 \\
\hline ko04141 & Protein processing in endoplasmic reticulum & 42 & 254 & 0.001695 & 7.15E-02 \\
\hline ko00280 & Valine, leucine and isoleucine degradation & 15 & 65 & 0.002402 & 8.45E-02 \\
\hline ko00062 & Fatty acid elongation in mitochondria & 6 & 16 & 0.004098 & $1.24 \mathrm{E}-01$ \\
\hline ko04612 & Antigen processing and presentation & 15 & 75 & 0.009839 & 2.60E-01 \\
\hline ko04146 & Peroxisome & 23 & 134 & 0.011384 & 2.67E-01 \\
\hline ko01040 & Biosynthesis of unsaturated fatty acids & 9 & 38 & 0.014408 & 2.67E-01 \\
\hline
\end{tabular}

Table 4. Differentially expressed transcripts validated by qRT-PCR analysis.

\begin{tabular}{|c|c|c|c|c|}
\hline \multirow[t]{2}{*}{ Gene ID } & \multirow[t]{2}{*}{ Description } & \multicolumn{2}{|c|}{ Fold Change* $^{*}$} & \multirow[t]{2}{*}{ SE } \\
\hline & & DGE & qRT-PCR & \\
\hline AG10278 & Flightin & 5961.13 & 1631.77 & 159.36 \\
\hline gi| 289097293 & Kat & 2068.00 & 11.06 & 1.93 \\
\hline AG4984 & Fabp & 1092.00 & 12.25 & 2.18 \\
\hline AG12533 & phosphate carrier protein, mitochondrial-like & 270.69 & 129.31 & 12.41 \\
\hline AG13078 & Ant & 52.62 & 55.20 & 0.78 \\
\hline AG11798 & very long-chain specific acyl-CoA dehydrogenase, mitochondrial-like & 11.63 & 12.14 & 2.33 \\
\hline AG10938 & SerRS & -837.00 & -3.32 & 0.21 \\
\hline AG13124 & ribosomal protein S28e-like & 11.45 & 26.07 & 5.96 \\
\hline AG13172 & ribosomal protein LP0 & -4.53 & -2.03 & 0.54 \\
\hline AG12143 & ribosomal protein S27A & -4.37 & -3.42 & 1.07 \\
\hline AG13161 & $40 S$ ribosomal protein S4-like & -4.15 & -1.18 & 0.17 \\
\hline AG11336 & $40 S$ ribosomal protein S21-like & -4.03 & -2.46 & 0.18 \\
\hline AG13187 & ribosomal protein L15 & -4.03 & -3.76 & 1.16 \\
\hline AG8620 & acylphosphatase & 11.18 & 26.64 & 6.96 \\
\hline gi| 289143076 & acylphosphatase-2-like & -9.64 & -2.06 & 0.46 \\
\hline AG8620 & pyruvate kinase-like & -7.07 & -1.87 & 0.69 \\
\hline AG10855 & malate dehydrogenase, cytoplasmic-like & -6.52 & -2.70 & 0.99 \\
\hline AG12539 & pyruvate dehydrogenase E1 component subunit alpha, mitochondrial-like & -4.16 & -2.51 & 1.21 \\
\hline AG9726 & proteasome activator complex subunit 3-like & -4.88 & -1.69 & 0.18 \\
\hline AG12585 & proteasome inhibitor-like & -4.32 & -1.10 & 0.05 \\
\hline
\end{tabular}






Figure 3. Gene ontology classification of transcripts differentially expressed between winged and wingless $A$. gossypii. A total of 763 genes ( $45.88 \%$ of the differentially expressed transcripts) were categorized into three Gene Ontology classes, cellular components, molecular functions, and biological processes, respectively.

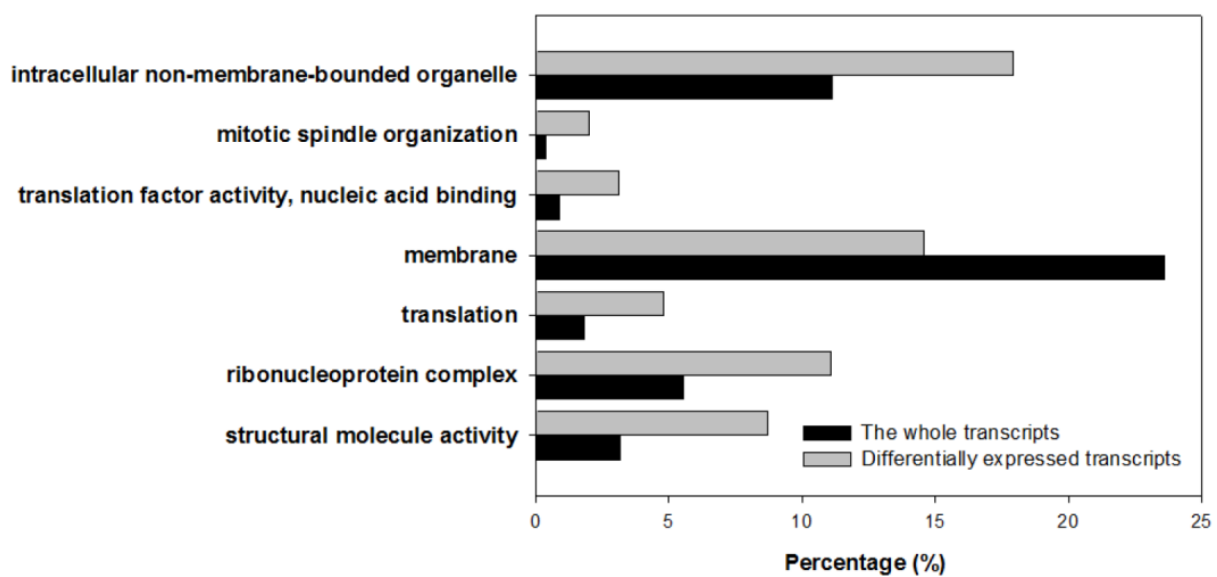

Figure 4. Differentially expressed transcripts enriched specific Gene Ontology terms. All 763 differentially expressed transcripts are enriched in 16 GO groups. 7 deep level (specific) GO terms were gained. 6 terms were overrepresented, and one term was underrepresented.

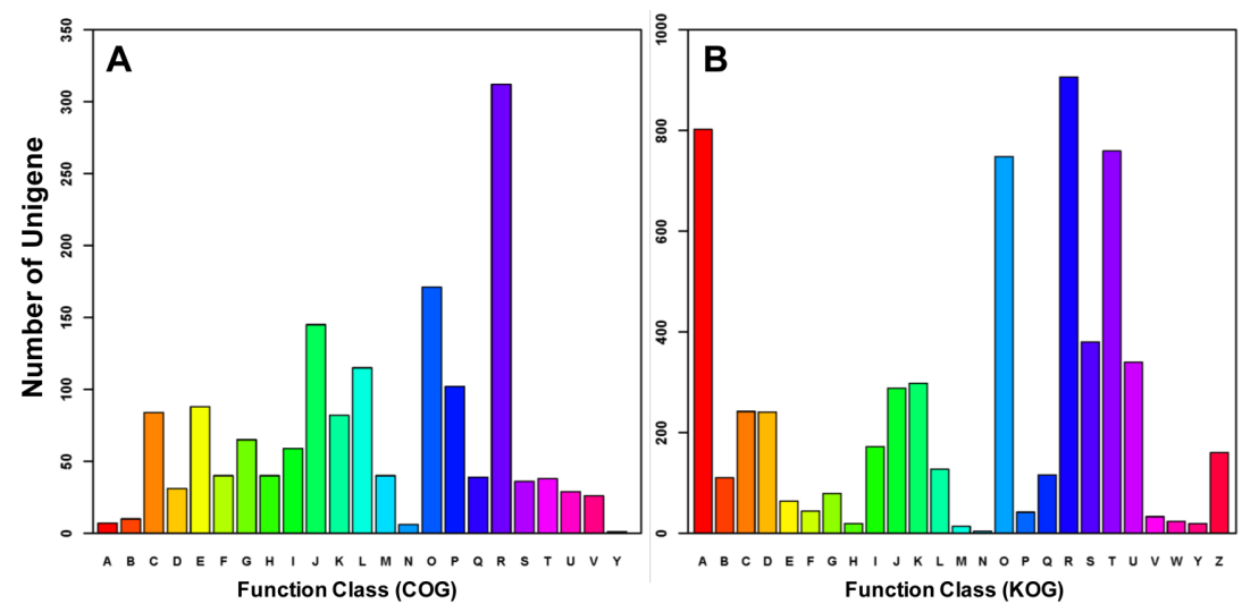

Figure 5. Functional classification of differentially expressed transcripts between winged and wingless $A$. gossypii. Based on Clusters of Orthologous Groups (COG, Figure 5A), 676 genes (40.65\% of the Differentially expressed transcripts ) were annotated and categorized into 23 functional classes. In comparison, I I 44 genes (68.79\% of the Differentially expressed transcripts ) were annotated and categorized into 25 specific classes within the euKaryotic Orthologous Groups (KOG, Figure 5B). A: RNA processing and modification, B: Chromatin structure and dynamics, C: Energy production and conversion, D: Cell cycle control, cell division, chromosome partitioning, E: Amino acid transport and metabolism, F: Nucleotide transport and metabolism, G: Carbohydrate transport and metabolism, $\mathrm{H}$ : Coenzyme transport and metabolism, I: Lipid transport and metabolism, J: Translation, ribosomal structure and biogenesis, K: Transcription, L: Replication, recombination and repair, M: Cell wall/membrane/envelope biogenesis, N: Cell motility, O: Posttranslational modification, protein turnover, chaperones, P: Inorganic ion transport and metabolism, Q: Secondary metabolites biosynthesis, transport and catabolism, R: General function prediction only, S: Function unknown, T: Signal transduction mechanisms, U: Intracellular trafficking, secretion, and vesicular transport, V: Defence mechanisms, W: Extracellular structures, Y: Nuclear structure, Z: Cytoskeleton. 


\section{Discussion}

\section{DGE analysis}

We sequenced wing-polyphenic aphids using the DGE approach and obtained 10.69 million raw base sequences, 10.48 million of which were clean tags. Clean tags were compared in different libraries. Interestingly, compared to the clean tags in non-specific forms, clean tags of morph-specific forms account for a small fraction of the total clean tags (Figure 1A), however, they comprise a higher level of percentage in distinct tags (Figure 1B), indicating that morph-specific tags have a high diversity. This result may suggest that expression level of common genes which maintain the basic growth and development is higher relative to specific genes that play crucial and multiple roles in trait variation. Typically, the reference sequences used by DGE analysis are selected from genome sequence information of evolutionarily related species to the target species, especially when there is no genome information available for the target species. In current DGE analysis, we chose EST sequence of $A$. gossypii as reference sequences, 46,766 tags were mapped to 18,141 transcripts (Table 1).

\section{Differentially expressed transcripts}

Through DGE analysis, we obtained a total of 1,663 differentially expressed transcripts, in which 58 transcripts were expressed significantly higher in winged aphids and 1,605 transcripts had a higher expression in wingless A. gossypii (Figure 2). This discrepancy is a reflection of the flight-reproduction trade-offs, in which genes associated with reproduction is likely over-numbered the flight capability. Among these differentially expressed transcripts, Flightin and seryl-tRNA synthetase (SerRS) was highly expressed in winged and wingless $A$. gossypii, respectively. Transcripts related to lipid metabolism and energy production were found at higher expression levels in migratory morphs (Table 2, Supplementary Material: Table S2). Differential energy allocation is an important part of trade-offs between flight capability and reproduction. This result is consistent with the previous observation of the significantly higher triglyceride content in winged aphid versus the wingless aphid [30, 31].

Associated with lipid synthesis and degradation, transcripts of 3-ketoacyl-CoA thiolase (KAT), very long-chain specific acyl-CoA dehydrogenase, medium-chain specific acyl-CoA dehydrogenase and long-chain-fatty-acid-CoA ligase 1 were shown significantly higher expression levels in winged morphs. Furthermore, 15 highly expressed "mitochondrial like" transcripts in winged morphs may contribute to energy production and thought to be involved in as- pects of the citric acid cycle, respiratory chain, ATP synthesis and transport. These results suggest that lipid is the fuel for aphid migration. Similarly, transcripts involved in energy production showed elevated transcriptions in winged morphs of A. pisum [34] and the brown planthopper, Nilaparvata lugens [40].

Differentially expressed transcripts were significantly enriched in 7 GO terms, and 5 of them, structural molecule activity, ribonucleoprotein complex, translation, translation factor activity/nucleic acid binding, mitotic spindle organization, were directly or indirectly associated with protein translation and assembly. This is consistent with the pathway enrichment analysis. The remaining 2 term, membrane and intracellular non-membrane-bounded organelle, were associated with organelle, in which a series of biological process occurs, including partial protein synthesis processes in endoplasmic reticulum, fatty acid elongate in mitochondria, peroxisome break down very long chain fatty acids.

qRT-PCR validation study confirmed the results from DGE analysis (Table 4). Fully replicated qRT-PCR analysis, to certain extent, complements the lack of DGE replications. All 20 genes subjected to the qRT-PCR analysis showed the similar trends with the DGE results. In comparison to DGE analysis, however, the fold changes in gene expression of Flightin, Fabp, Kat and SerRS were less dramatic in qRT-PCR analysis. Such discrepancy is not without precedent [40]. Due to the computation needs (the lowest expression level of any gene in the DGE analysis, by default, is 0.01 even if it is not expressed), the differential gene expression level is typically overestimated in the DGE analysis. In this study, the expression of Fabp, Kat and SerRS were morph-specific, and consequently, the result from the DGE analysis is likely overestimated.

\section{Pathway enrichment analysis}

Through the Kyoto Encyclopedia of Genes and Genomes (KEGG) annotation system, differentially expressed transcripts were significantly enriched $(\mathrm{Q}<0.05)$ in three pathways, ribosome, pyruvate metabolism and proteasome (Table 3). Of the differentially expressed transcripts $5.58 \%$ were in the ribosome pathway. While in the whole reference background, only $1.72 \%$ of transcripts were annotated in this pathway. The ribosome is required for protein synthesis, which is critical for all aspects of cell growth and division [49]. They are usually recognized as stable expression genes. However, in the current study, 48 highly expressed transcripts in wingless morph and 1 in winged morph were coded for the ribosomal proteins. Ribosomal genes are typically 
highly expressed genes. Previously reported research results showed gene products that are components of ribosomes were over-representation in A. pisum [34]. One $40 S$ ribosomal protein S20 displayed a higher expression level in brachypterous morph of $N$. lugens than in macropterous adult females [40]. This evidence convinces us of difference in ribosomal proteins between two morphs of A. gossypii. Our data indicated that more protein synthesis was detected in the non-dispersing morphs, which results in faster developmental rates and higher fecundity $[14,50]$.

It is expected that pyruvate metabolism was a differentially expressed transcripts enriched pathway. 21 transcripts in this pathway were screened as differentially expressed transcripts. Pyruvate is a key intersection in several metabolic pathways, and known as the "hub" of carbohydrate, fats and proteins $[16,46]$. Pyruvate can be generated from glucose through glycolysis [16]. In recent study, wingless morphs showed significantly higher soluble sugar and glycogen content than winged morphs during adulthood in A. gossypii [30] and in S. avenae [31]. The result of gene expression was consistent with our previous research in biochemical composition. Most transcripts in this pathway were highly expressed in wingless morphs. Our results suggest that sugar or glycogen metabolism is faster in wingless morphs and those carbohydrates were used for development and production. This is consistent with the contention that sugar and glycogen is not the fuel of aphids for migration [51].

$26 \mathrm{~S}$ proteasome, working with ubiquitin, operates the energy-dependent regulated proteolysis process in eukaryotic cells. The immunotype proteasomes, whose catalytic subunits are replaced by the related counterparts, were discovered recently in vertebrates [52]. The protein degradation process including immunity may be diverse in different morphs. In previous study, soluble protein content were higher in dispersal morphs than in reproduction morphs in A. gossypii [30] and S. avenae [31]. As such, proteasome for protein degradation and protein turnover increased.

\section{Genes of interests}

Genes which we are interested in include Flightin, fatty acid binding protein gene (Fabp), adenine nucleotide translocase gene (Ant), abnormal wing discs1 (Awd1) and abnormal wing discs2 (Awd2) (Supplementary Material: Table S2).

The primary difference between winged and wingless adults is the existence of flight apparatus, including wing and indirect flight muscle. For winged adults, genes expressed in muscle were identified in this study, Flightin and Fabp. Flightin is a multiply phosphorylated, myofibrillar protein in Drosophila indirect flight muscles (IFM), and an essential part of the flight muscle contractile mechanism for thick filament assembly and sarcomere stability in Drosophila [53-56]. Particularly, Flightin exhibits the greatest differential transcript accumulation in winged morphs of A. pisum [34] and N. lugens [40]. Another gene coding FABP in muscle was one of the top ten most differentially expressed genes in winged morphs. FABP will increase the solubility of fatty acids and thus lead to a rapid transport through the cytosol, since the lipid is the preferred fuel for insect migrating [57] In recent research, free fatty acid content was significantly higher in winged than wingless adults before flight behavior in A. gossypii [30] and A. pisum [31].

ANT belongs to the mitochondria carrier protein which is embedded in the inner membrane of mitochondria and required for importing ADP into the mitochondrial matrix throughout the ATP synthesis [33]. The gene expression of Ant gene is significantly higher in winged morphs than wingless morphs in $A$. gossypii (Table 2). The expression pattern of Ag Ant is similar as that of $M p$ Ant [33], suggesting the need of energy for winged morphs during the radiation to diverse food resources and habitats [58].

The awd1 and awd2 showed a significantly higher expression level in wingless morphs than winged morphs in A. gossypii. The awd of Drosophila encodes a nucleoside diphosphate kinase (NDPK). Complete loss of function of the awd gene causes lethality in Drosophila after the larval stage. Mutations in awd gene cause abnormal tissue morphology and aberrant differentiation. [59, 60]. The awd plays an important role in development and differentiation of insects. They are not involved in the presumptive wing-patterning gene network [61]. Little attention was given to awd genes in the previous studies related to wing polyphenism.

\section{Perspectives and future research}

This study sheds lights on the genetic basis of trade-offs between flight capability and reproduction. Genes involved in lipid metabolism show higher expression levels in winged $A$. gossypii than wingless morphs. In contrast, genes involved in sugar metabolism are elevated in wingless morphs. Our combined results suggest aphids using lipid rather than sugar or glycogen as fuel during migration. Flight ability of alate aphids depends on the energy supply of lipids. $\mathrm{KAT}, \mathrm{FABP}$, and very long-chain specific acyl-CoA dehydrogenase seems to be more important for winged morphs. Silencing of these genes using RNA interference (RNAi) may disrupt their energy supply, and compromise their ability to fly. RNAi through injection [62, 63] and feeding [64] methods have been 
well established in A. pisum. It is germane to investigate the pest control potential of these genes, including Flightin, through genetically interrupting the dispersal of A. gossypii.

\section{Supplementary Material}

Table S1-S4, and Fig.S1 - S2.

http://www.ijbs.com/v10p0257s1.xlsx

\section{Abbreviations}

DGE: Digital gene expression; EST: Expressed Sequence Tag; TPM: Number of transcripts per million clean tags; FDR: False Discovery Rate; GO: Gene Ontology; COG: Cluster of Orthologous Groups; KEGG: Kyoto Encyclopedia of Genes and Genomes; KO: KEGG ontology; KOG: euKaryotic Orthologous Groups; qRT-PCR: Quantitative real-time PCR, IFM: indirect flight muscles; SerRS: seryl-tRNA synthetase; KAT: 3-ketoacyl-CoA thiolase; FABP: fatty acid binding protein; NDPK: nucleoside diphosphate kinase; ANT: adenine nucleotide translocase.

\section{Acknowledgements}

Special thanks go to Timothy Moural (University of Washington State) and Drs. Tracy Lu and John Obrycki (University of Kentucky) for their comments on an earlier version and Wenbo Chen for his assistance in the data analysis. This research was supported by the National Science \& Technology Pillar Program (2012BAD19B05). Xiaowei Yang was supported by a start-up fund to Zhou and College of Agriculture, University of Kentucky. The granting agencies have no role in study design, data collection and analysis, decision to publish, or preparation of the manuscript.

\section{Data Deposition}

The accession numbers for DGE libraries derived from sedentary and migratory morphs of cotton aphids, Aphis gossypii, are SRX246107 and SRX242773, respectively.

\section{Competing Interests}

The authors have declared that no competing interest exists.

\section{References}

1. Stearns SC. Trade-offs in life-history evolution. Functional ecology. 1989; 3: 259-68.

2. Roff DA. Evolution of life histories: theory and analysis. New York, USA: Chapman \& Hall; 1992.

3. Stearns SC. The evolution of life histories. New York, USA: Oxford University Press; 1992.

4. Brackenbury J. Insects in flight. London, UK: Blandford; 1992

5. Moller Andersen N. Seasonal polymorphism and developmental changes in organs of flight and reproduction in bivoltine pondskaters (Hem. Gerridae). Insect Systematics \& Evolution. 1973; 4: 1-20.
6. Gunn A, Gatehouse A, Woodrow K. Trade-off between flight and reproduction in the African armyworm moth, Spodoptera exempta. Physiological Entomology. 1989; 14: 419-27.

7. Kullberg C, Houston DC, Metcalfe NB. Impaired flight ability-a cost of reproduction in female blue tits. Behavioral Ecology. 2002; 13: 575-9.

8. Zera AJ, Denno RF. Physiology and ecology of dispersal polymorphism in insects. Annu Rev Entomol. 1997; 42: 207-30.

9. Guerra PA. Evaluating the life-history trade-off between dispersal capability and reproduction in wing dimorphic insects: a meta-analysis. Biological reviews of the Cambridge Philosophical Society. 2011; 86: 813-35.

10. Roff DA. The Evolution of Wing Dimorphism in Insects. Evolution. 1986; 40: 1009-20.

11. Harrison RG. Dispersal Polymorphisms in Insects. Annual Review of Ecology and Systematics. 1980; 11: 95-118.

12. Wang ZL, Liu TT, Huang ZY, Wu XB, Yan WY, Zeng ZJ. Transcriptome Analysis of the Asian Honey Bee Apis cerana cerana. PLoS One. 2012; 7: e47954.

13. Roff DA. The Evolution of Wing Dimorphism in Insects. Evolution. 1986; 40: 1009 .

14. Braendle C, Davis GK, Brisson JA, Stern DL. Wing dimorphism in aphids. Heredity (Edinb). 2006; 97: 192-9.

15. Castañeda LE, Figueroa CC, Bacigalupe LD, Nespolo RF. Effects of wing polyphenism, aphid genotype and host plant chemistry on energy metabolism of the grain aphid, Sitobion avenae. Journal of Insect Physiology. 2010; 56: 1920-4.

16. Simpson SJ, Sword GA, Lo N. Polyphenism in insects. Current Biology. 2011; 21: R738-R49.

17. Xu X, Liu X, Zhang $\mathrm{Q}, \mathrm{Wu}$ J. Morph-specific differences in life history traits between the winged and wingless morphs of the aphid, Sitobion avenae (Fabricius) (Hemiptera: Aphididae). Entomol Fennica 2011; 22: 56-64.

18. Zera AJ. Wing polymorphism in Gryllus (Orthoptera: Gryllidae): Proximate endocrine, energetic and biochemical mechanisms underlying morph specialization for flight vs. reproduction. Phenotypic Plasticity of Insects: Mechanisms and Consequences. 2009: 609-53.

19. Tanaka S, Nishide Y. First Record of the Occurrence and Genetics of a Short-Winged Morph in the Migratory Locust,Locusta migratoria(Orthoptera: Acrididae). Journal of Orthoptera Research. 2012; 21: 169-74.

20. Zera AJ, Sall J, Otto K. Biochemical aspects of flight and flightlessness in Gryllus: flight fuels, enzyme activities and electrophoretic profiles of flight muscles from flight-capable and flightless morphs. J Insect Physiol. 1999; 45: 275-85.

21. Zhao Z, Zera AJ. Differential lipid biosynthesis underlies a tradeoff between reproduction and flight capability in a wing-polymorphic cricket. Proceedings of the National Academy of Sciences of the United States of America. 2002; 99: 16829-34.

22. Zera AJ, Zhao Z. Intermediary metabolism and life-history trade-offs: differential metabolism of amino acids underlies the dispersal-reproduction trade-off in a wing-polymorphic cricket. Am Nat. 2006; 167: 889-900.

23. Müller CB, Williams IS, Hardie J. The role of nutrition, crowding and interspecific interactions in the development of winged aphids. Ecological Entomology. 2001; 26: 330-40.

24. Braendle C, Friebe I, Caillaud MC, Stern DL. Genetic variation for an aphid wing polyphenism is genetically linked to a naturally occurring wing polymorphism. Proceedings Biological sciences / The Royal Society. 2005; 272: 657-64.

25. Simon JC, Rispe C, Sunnucks P. Ecology and evolution of sex in aphids. Trends in Ecology \& Evolution. 2002; 17: 34-9.

26. Moran NA. The Evolution of Aphid Life Cycles. Annual Review of Entomology. 1992; 37: 321-48.

27. Simpson SJ, Sword GA, Lo N. Polyphenism in insects. Current biology: CB. 2011; 21: R738-49.

28. Bhatia V, Uniyal PL, Bhattacharya R. Aphid resistance in Brassica crops: challenges, biotechnological progress and emerging possibilities. Biotechnol Adv. 2011; 29: 879-88.

29. Ishikawa A, Hongo S, Miura T. Morphological and histological examination of polyphenic wing formation in the pea aphid Acyrthosiphon pisum (Hemiptera, Hexapoda). Zoomorphology. 2008; 127: 121-33.

30. Shi SL, Liu XX, Zhang QW, Zhao ZW. Morph-specific differences in metabolism related to flight in the wing-dimorphic Aphis gossypii. Insect Science. 2010; 17: 527-34.

31. Xu XL, Liu XX, Zhang QW, Wu JX. Morph-specific differences in biochemical composition related to flight capability in the wing-polyphenic Sitobion avenae. Entomologia Experimentalis Et Applicata. 2011; 138: 128-36.

32. Brisson JA. Aphid wing dimorphisms: linking environmental and genetic control of trait variation. Philosophical transactions of the Royal Society of London Series B, Biological sciences. 2010; 365: 605-16.

33. Ghanim M, Dombrovsky A, Raccah B, Sherman A. A microarray approach identifies ANT, OS-D and takeout-like genes as differentially regulated in alate and apterous morphs of the green peach aphid Myzus persicae (Sulzer). Insect Biochem Mol Biol. 2006; 36: 857-68.

34. Brisson JA, Davis GK, Stern DL. Common genome-wide patterns of transcript accumulation underlying the wing polyphenism and polymorphism in the pea aphid (Acyrthosiphon pisum). Evol Dev. 2007; 9: 338-46.

35. International Aphid Genomics C. Genome sequence of the pea aphid Acyrthosiphon pisum. PLoS Biol. 2010; 8: e1000313. 
36. Xiao S, Mo D, Wang Q, Jia J, Qin L, Yu X, et al. Aberrant host immune response induced by highly virulent PRRSV identified by digital gene expression tag profiling. BMC Genomics. 2010; 11: 544.

37. $t$ Hoen PA, Ariyurek Y, Thygesen HH, Vreugdenhil E, Vossen RH, de Menezes RX, et al. Deep sequencing-based expression analysis shows major advances in robustness, resolution and inter-lab portability over five microarray platforms. Nucleic Acids Res. 2008; 36: e141.

38. Kundu R, Dixon AFG. Evolution of Complex Life-Cycles in Aphids. Journal of Animal Ecology. 1995; 64: 245-55.

39. Harhay GP, Smith TP, Alexander LJ, Haudenschild CD, Keele JW, Matukumalli LK, et al. An atlas of bovine gene expression reveals novel distinctive tissue characteristics and evidence for improving genome annotation. Genome Biol. 2010; 11: R102.

40. Xue J, Bao YY, Li BL, Cheng YB, Peng ZY, Liu H, et al. Transcriptome analysis of the brown planthopper Nilaparvata lugens. PLoS One. 2010; 5: e14233.

41. Liu F, Li W, Li Z, Zhang S, Chen S, Su S. High-abundance mRNAs in Apis mellifera: comparison between nurses and foragers. J Insect Physiol. 2011; 57: 274-9.

42. Audic S, Claverie JM. The significance of digital gene expression profiles. Genome Res. 1997; 7: 986-95.

43. Yoav B, Daniel Y. The control of the false discovery rate in multiple testing under dependency. The Annals of Statistics. 2001; 29: 1165-88.

44. Rosengard AM, Krutzsch HC, Shearn A, Biggs JR, Barker E, Margulies IMK, et al. Reduced $\mathrm{Nm} 23$ /Awd protein in tumour metastasis and aberrant Drosophila development. Nature. 1989; 342: 177-80.

45. Tatusov RL. A Genomic Perspective on Protein Families. Science. 1997; 278: 631-7.

46. Tatusov RL, Fedorova ND, Jackson JD, Jacobs AR, Kiryutin B, Koonin EV, et al. The COG database: an updated version includes eukaryotes. BMC bioinformatics. 2003; 4: 41 .

47. Conesa A, Gotz S, Garcia-Gomez JM, Terol J, Talon M, Robles M. Blast2GO: a universal tool for annotation, visualization and analysis in functional genomics research. Bioinformatics. 2005; 21: 3674-6.

48. Kanehisa M, Araki M, Goto S, Hattori M, Hirakawa M, Itoh M, et al. KEGG for linking genomes to life and the environment. Nucleic Acids Res. 2008; 36: D480-4.

49. Lin JI, Mitchell NC, Kalcina M, Tchoubrieva E, Stewart MJ, Marygold SJ, et al. Drosophila ribosomal protein mutants control tissue growth non-autonomously via effects on the prothoracic gland and ecdysone. PLoS Genet. 2011; 7: e1002408.

50. Schwartzberg EG, Kunert G, Westerlund SA, Hoffmann KH, Weisser WW. Juvenile hormone titres and winged offspring production do not correlate in the pea aphid, Acyrthosiphon pisum. J Insect Physiol. 2008; 54: 1332-6.

51. Dixon AFG, Kindlmann P. Cost of Fligth Apparatus and Optimum Body Size of Aphid Migrants. Ecology. 1999; 80: 1678-90.

52. Tanaka K, Mizushima T, Saeki Y. The proteasome: molecular machinery and pathophysiological roles. Biological Chemistry. 2012; 393(4):217-34.

53. Vigoreaux JO, Hernandez C, Moore J Ayer G, Maughan D. A genetic deficiency that spans the flightin gene of Drosophila melanogaster affects the ultrastructure and function of the flight muscles. Journal of Experimental Biology. 1998; 201: 2033-44.

54. Vigoreaux JO, Saide JD, Valgeirsdottir K, Pardue ML. Flightin, a novel myofibrillar protein of Drosophila stretch-activated muscles. The Journal of Cell Biology. 1993; 121: 587-98.

55. Reedy MC, Bullard B, Vigoreaux JO. Flightin Is Essential for Thick Filament Assembly and Sarcomere Stability in Drosophila Flight Muscles. The Journal of Cell Biology. 2000; 151: 1483-500.

56. Ayer G, Vigoreaux J. Flightin is a myosin rod binding protein. Cell Biochem Biophys. 2003; 38: 41-54.

57. Haunerland NH. Transport and utilization of lipids in insect flight muscles*. Comparative Biochemistry and Physiology Part B: Biochemistry and Molecular Biology. 1997; 117: 475-82.

58. Santamaria M, Lanave $C$, Saccone $C$. The evolution of the adenine nucleotide translocase family. Gene. 2004; 333: 51-9.

59. Timmons L, Shearn A. Role of AWD/nucleoside diphosphate kinase in Drosophila development. J Bioenerg Biomembr. 2000; 32: 293-300.

60. Dearolf CR, Hersperger E, Shearn A. Developmental consequences of awdb3, a cell-autonomous lethal mutation of Drosophila induced by hybrid dysgenesis. Developmental Biology. 1988; 129: 159-68.

61. Brisson JA, Ishikawa A, Miura T. Wing development genes of the pea aphid and differential gene expression between winged and unwinged morphs. Insect Mol Biol. 2010; 19 Suppl 2: 63-73.

62. Mutti NS, Park Y, Reese JC, Reeck GR. RNAi knockdown of a salivary transcript leading to lethality in the pea aphid, Acyrthosiphon pisum. Journal of insect science (Online). 2006; 6: 1-7.

63. Jaubert-Possamai S, Le Trionnaire G, Bonhomme J, Christophides GK, Rispe C, Tagu D. Gene knockdown by RNAi in the pea aphid Acyrthosiphon pisum. BMC biotechnology. 2007; 7: 63.

64. Pitino M, Coleman AD, Maffei ME, Ridout CJ, Hogenhout SA. Silencing of aphid genes by dsRNA feeding from plants. PloS one. 2011; 6: e25709. 